\title{
The importance of imaging methods in catheter dysfunction: Two rare cases and their management
}

\author{
Ersin Çelik ${ }^{1}$, Ahmet Çora ${ }^{1} \mathbb{D}$, Kadir Burhan Karadem² ${ }^{\mathbb{D}}$ \\ ${ }^{1}$ Department of Cardiovascular Surgery, Isparta City Hospital, Isparta, Turkey \\ ${ }^{2}$ Department of Cardiovascular Surgery, Süleyman Demirel University, Faculty of Medicine, Isparta, Turkey
}

\begin{abstract}
Cuffed-tunneled hemodialysis catheter is a reliable and effective alternative treatment option in the cases where all the peripheral vascular access points become useless for operative procedures or reinterventions. In addition, imaging methods have become more important to identify or prevent possible complications during or after catheter insertion. In this article, we report two rare cases to emphasize the importance of fluoroscopy and venography in determination and treatment of catheter-related complications and dysfunctions.
\end{abstract}

Keywords: Catheter dysfunction, cuffed-tunneled hemodialysis catheter, fluoroscopy, hemodialysis insufficiency, venography.

Cuffed-tunneled hemodialysis catheter is a reliable and effective alternative treatment option for end-stage renal disease, during which all the peripheral vascular access points become useless for operative procedures or reinterventions. However, infection, thrombosis, cardiac arrhythmia, entrapped guidewire, and malposition of the catheter are among the complications that are possible to be faced during or after a catheter insertion. ${ }^{[1]}$ Yet, malposition of the hemodialysis catheter tip into the hepatic veins and inferior vena cava (IVC) occlusion are rare complications which may be seen after the operative procedures. ${ }^{[2]}$

In this article, we report two rare cases to emphasize the importance of fluoroscopy and venography in determination and treatment of catheter-related complications and dysfunctions.

\section{CASE REPORT}

Case 1- A 40-year-old female patient who was on chronic hemodialysis treatment for three times per week for several years was consulted. The patient had many failed operations in both upper extremities with the purpose of creating a vascular access point. Her medical history revealed the placement of a cuffed-tunneled hemodialysis catheter (14.5-Fr, 32-cm; Palindrome Chronic Catheter, Covidien AG, Dublin, Ireland) into the right internal jugular vein (IJV), which was applied by another surgeon in an external clinic without fluoroscopy due to the lack of peripheral access approximately a week ago. The patient had no clinical complaints; however, despite dialysis for three days a week, serum creatine, alanine aminotransferase (ALT), and aspartate aminotransferase (AST) levels were found to be elevated. Based on the chest X-ray and thoracic computed tomography (CT) imaging, malposition of the catheter tip to the middle hepatic vein was detected (Figure 1).

Under the guidance of fluoroscopy, the catheter was replaced with a shorter one $(14.5-\mathrm{Fr}, 28-\mathrm{cm}$; Palindrome Chronic Catheter, Covidien AG, Dublin, Ireland). On fluoroscopy, the placement of the catheter tip was confirmed, as it was in the right 

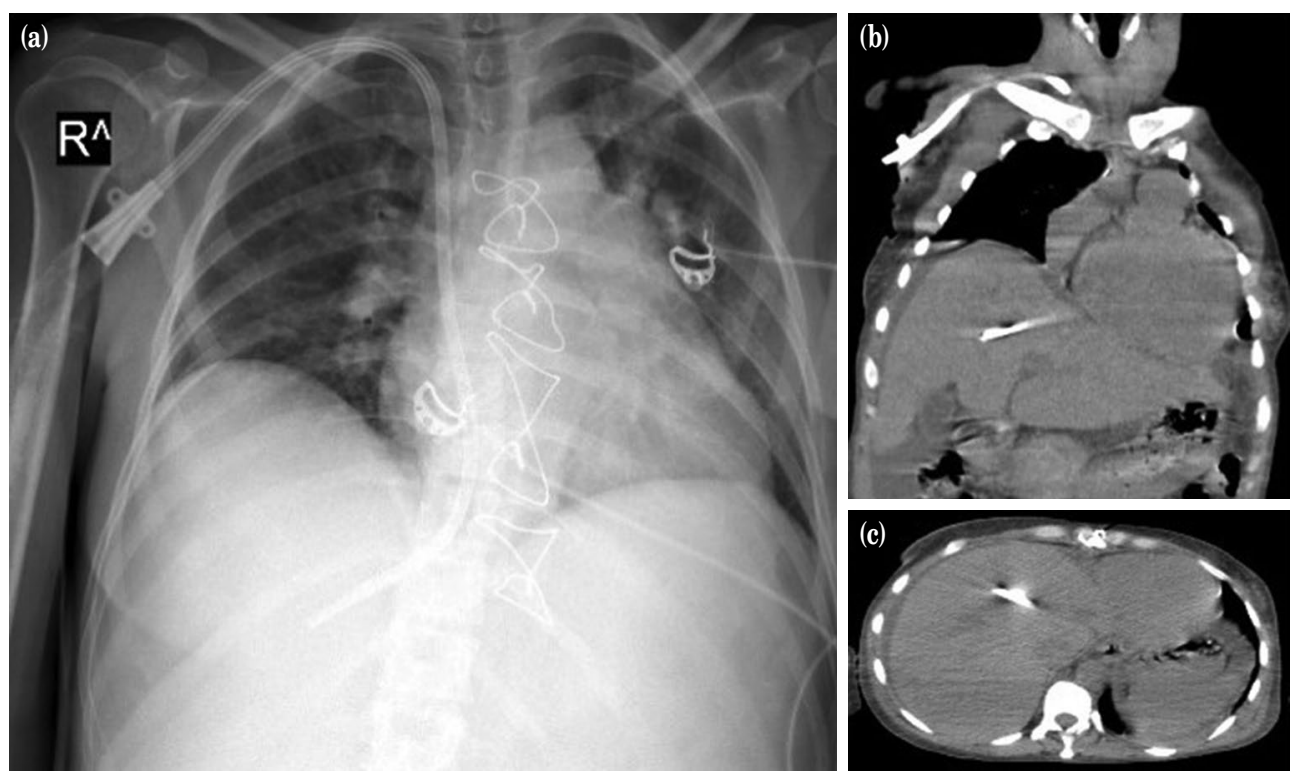

Figure 1. Malposition of the cuffed-tunneled hemodialysis catheter. (a) View of catheter malposition on chest X-ray. (b, c) View of catheter malposition into the middle hepatic vein on computed tomography.

atrium. The placement of the catheter tip and possible pneumothorax were controlled with chest X-ray. The patient went on the hemodialysis therapy uneventfully and, after the operation, a progressive decline in the biochemical parameters was observed.

A written informed consent was obtained from the patient for publication of this case report and any accompanying images.

Case 2- A 55-year-old male patient with diabetes who was on chronic hemodialysis treatment for three times per week for 20 years was consulted. Due to the lack of peripheral access, hemodialysis treatment was applied via a cuffed-tunneled hemodialysis catheter for two years. To correct dysfunction of the catheter, his cuffed-tunneled catheter located in the right IJV was placed into the right femoral vein $(\mathrm{FV})$, which was accessed via a cuffed-tunneled catheter about eight months ago. Two weeks ago, the cuffed-tunneled catheter in the right $\mathrm{FV}$ was removed due to the catheter dysfunction caused by another center. The right IJV puncture was performed; however, the guidewire was unable to be advanced. Therefore, another cuffed-tunneled hemodialysis catheter (14.5-Fr, 32-cm, Palindrome Chronic Catheter, Covidien AG, Dublin, Ireland) was placed in the left
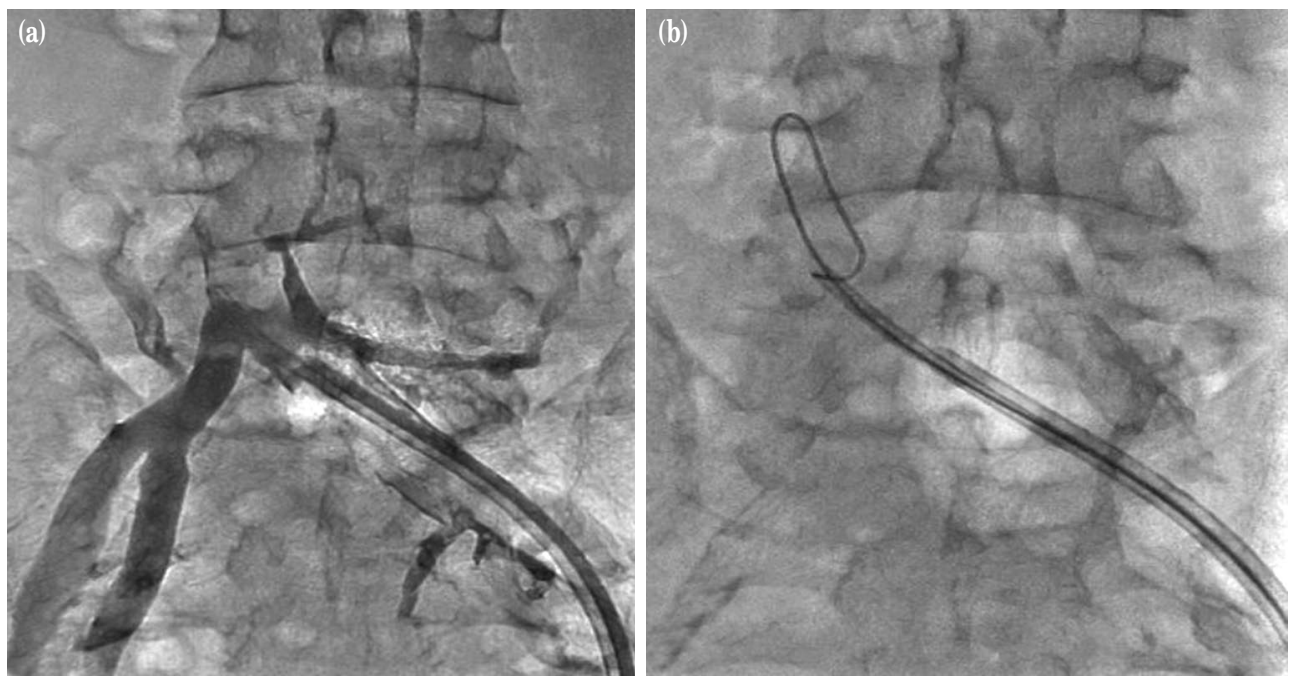

Figure 2. Thrombosis of distal inferior vena cava. (a) Inferior vena cavography. (b) Non-advance hydrophilic guide wire. 

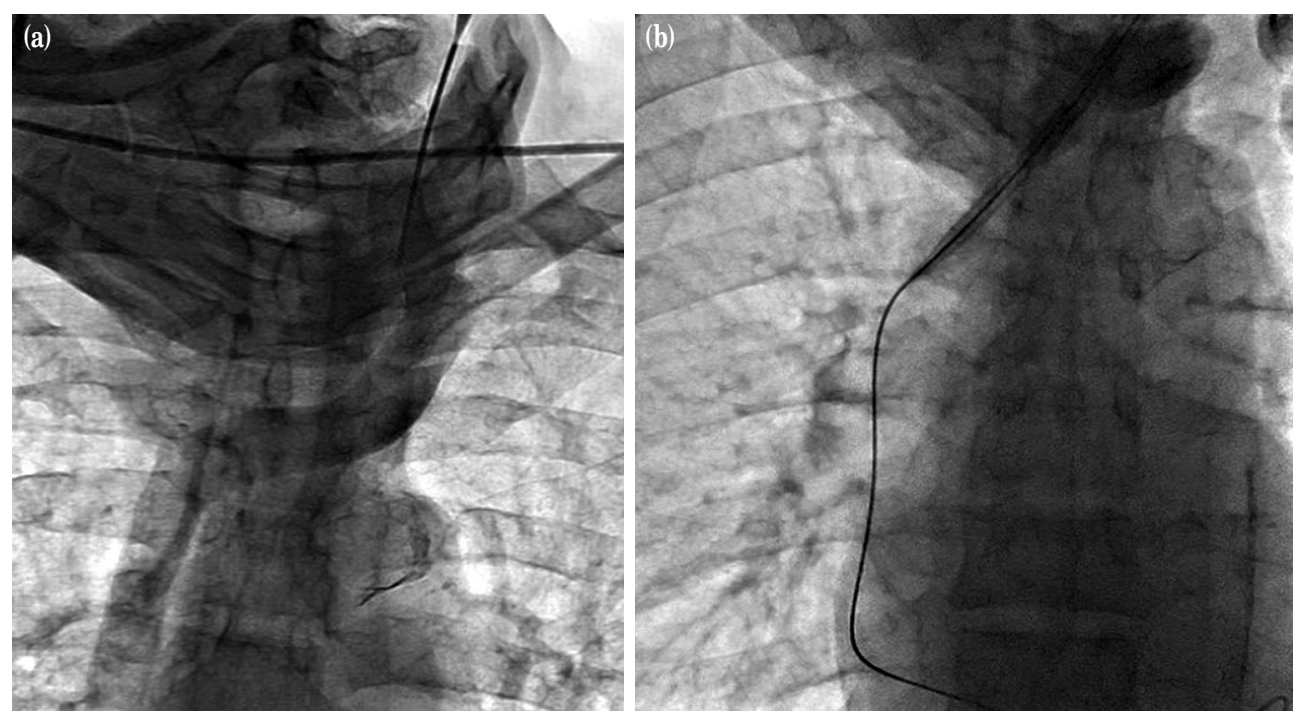

Figure 3. (a) Venography from the left internal jugular vein (b) view of guidewire and cuffed-tunneled catheter

FV. On the final hemodialysis treatment, the patient was evaluated regarding inadequate flow from both lumens of the new catheter. Venography was performed via the left femoral tunneled catheter to determine the problem, and total thrombosis just proximal to iliac bifurcation was observed (Figure 2a). After failing an attempt to pass the occluded part through the IVC by a hydrophilic guidewire, the intervention for IVC thrombosis was terminated (Figure 2b). For generalized ecchymosis and hematoma on the right side of the neck, left IJV puncture was performed under the ultrasonographic guidance. Fluoroscopic imaging was made via a puncture needle and no occlusion was detected on the left IJV, brachiocephalic vein, or VCS (Figure 3a). A cuffed-tunneled hemodialysis catheter 14.5-Fr, 32-cm; Palindrome Chronic Catheter, Covidien AG, Dublin, Ireland) was placed with fluoroscopy guidance (Figure $3 \mathrm{~b}$ ).

A written informed consent was obtained from the patient for publication of this case report and any accompanying images.

\section{DISCUSSION}

The IJV, FV, or IVC can be used for the placement of permanent double-lumen hemodialysis catheters. Malposition of cuffed-tunneled hemodialysis catheter may cause ineffective dialysis, and even fatal outcomes. ${ }^{[1,3]}$

Malposition of the jugular or subclavian doublelumen central venous catheter is not an uncommon complication. The National Kidney Foundation Kidney
Disease Outcomes Quality Initiative (NKF-DOQI) guidelines recommend routine chest $\mathrm{X}$-ray to detect potential complications and to confirm the placement of the catheter after jugular and subclavian catheter insertion. ${ }^{[4]}$ Therefore, catheter malposition and subcutaneous traces must be checked through a chest $\mathrm{X}$-ray or fluoroscopy, and immediate repositioning of the catheter must be performed, if needed. In addition, the utilization of venography using a catheter can be helpful to determine the cause of the catheter dysfunction.

The right innominate vein, azygos vein, and internal thoracic vein are the most reported locations for malposition. Malposition can be seen as a result of selecting a too long catheter or inserting it too far into venous area, which is particularly more frequent when the operator does not use fluoroscopy. It can be also seen as a result of migration via inspiration-expiration movements or heart beats. Malposition of the cuffed-tunneled catheter into the hepatic vein as in the first case has rarely been reported in the literature. ${ }^{[2,5]}$

The cuffed-tunneled catheters are made of silicon or polyurethane materials. These materials reduce thrombogenicity and endothelial damage. As in our second case, they may cause thrombosis, even in the largest veins in terms of diameter. Although thrombosis or vascular damage is rarely seen even in misplaced dialysis catheters, symptoms or complications can develop depending on where the catheter is positioned after the insertion. As in the first case, due to the malposition into the hepatic 
vein, the lack of sufficient flow from the catheter and insufficient dialysis therapy can be observed, and chest pain or abdominal discomfort or pain may be seen as the manifestations in these patients. ${ }^{[2,5]}$ Also, hepatic vein injury and, after injury, a life-threatening complication such as hepatic vein thrombosis can be seen as a complication of this malposition. ${ }^{[5]}$ In the first case, only the lack of catheter flow on dialysis, insufficient hemodialysis and elevated AST and ALT levels were evaluated. After an immediate insertion of a new catheter into a suitable position, the patient's laboratory tests recovered rapidly and uneventfully. Either there is hemodialysis insufficiency or not, in the presence of elevated liver enzymes and atypical chest findings or abdominal pain occurred after placement of a dialysis catheter, malposition of the catheter into the hepatic vein must be kept in mind, as well as the other causes that could result in catheter dysfunction.

As in our second case, venography can be done via a present hemodialysis catheter to determine the problems such as pericatheter sleeve, thrombus formation or central venous occlusion, before changing the dysfunctional catheter. ${ }^{[6]}$ By applying the venography, we determined the IVC occlusion and we avoided multiple punctures to IVC. Additionally, venography via IJV puncture and determining the persistence of the left IJV and vena cava inferior, we apply the guidewire and place the cuffed-tunneled catheter to the left IJV safely without any complication.

In conclusion, whether for the first catheter placement attempt or for changing a dysfunctional catheter, we believe that fluoroscopy guidance, direct catheter venography, or puncture needle venography are particularly valuable diagnostic tools to identify or avoid possible complications that may occur during or after hemodialysis catheter placements.

\section{Declaration of conflicting interests}

The authors declared no conflicts of interest with respect to the authorship and/or publication of this article.

\section{Funding}

The authors received no financial support for the research and/or authorship of this article.

\section{REFERENCES}

1. Özbudak E, Yavuz Ş, Akgül A, Arıkan AA, Durmaz D, Şahin D, et al. Kalıcı hemodiyaliz kateterleri: Ne kadar kalıcı? Turk Gogus Kalp Dama 2013;21:646-53.

2. Yucel SK, Gumusel C. Malposition of hemodialysis catheter into the hepatic veins: A case report. Pak J Med Sci 2019;35:583-5.

3. Chen IC, Yang SC, Liu KT, Wu YH. Delayed malposition of a double-lumen hemodialysis catheter that caused hemorrhage and hypovolemic shock: A case report. Medicine (Baltimore) 2019;98:e14192.

4. III. NKF-K/DOQI clinical practice guidelines for vascular access: Update 2000. Am J Kidney Dis 2001;37(1 Suppl 1):S137-81.

5. Husainy MA, Chhina R, Alchanan R, Peddu P. Fatal outcome of haemodialysis catheter malposition into the hepatic vein causing Budd-Chiari Syndrome. J Vasc Access 2017;18:e37-e38.

6. Oguzkurt L, Tercan F, Torun D, Yildirim T, Zümrütdal A, Kizilkilic O. Impact of short-term hemodialysis catheters on the central veins: A catheter venographic study. Eur J Radiol 2004;52:293-9. 